Draft Version November 1, 2018

Preprint typeset using $\mathrm{LAT}_{\mathrm{E} X} \mathrm{X}$ style emulateapj v. 11/12/01

\title{
DEPENDENCE OF DUST OBSCURATION ON STAR FORMATION RATES IN GALAXIES
}

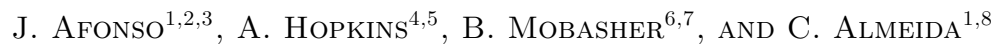 \\ Draft version November 1, 2018
}

\begin{abstract}
Many investigations of star formation rates (SFRs) in galaxies have explored details of dust obscuration, with a number of recent analyses suggesting that obscuration appears to increase in systems with high rates of star formation. To date these analyses have been primarily based on nearby $(z \leq 0.03)$ or UV selected samples. Using $1.4 \mathrm{GHz}$ imaging and optical spectroscopic data from the Phoenix Deep Survey, the SFR-dependent obscuration is explored. The use of a radio selected sample shows that previous studies exploring SFR-dependent obscurations have been biased against obscured galaxies. The observed relation between obscuration and SFR is found to be unsuitable to be used as an obscuration measure for individual galaxies. Nevertheless, it is shown to be successful as a first order correction for large samples of galaxies where no other measure of obscuration is available, out to intermediate redshifts $(z \approx 0.8)$.
\end{abstract}

Subject headings: galaxies: evolution — galaxies: starburst — radio continuum: galaxies

\section{INTRODUCTION}

Dust obscuration is currently recognized as one of the most serious sources of uncertainty in studies of galaxy evolution. With the recent results of far-infrared (FIR) and sub-mm observations revealing an ever increasing number of dusty star forming galaxies (e.g., Genzel \& Cesarsky 2000; Ivison et al. 2000; Smail et al. 2002), the need to unify measures of star formation rate (SFR) from independent indicators at different wavelengths (e.g., UV, $\mathrm{H} \alpha$, FIR, radio continuum) is as pressing as ever.

A relatively simple prescription for dust extinction correction to SFR has been suggested by Hopkins et al. (2001) and Sullivan et al. (2001), by assuming a luminosity- (or SFR) dependent obscuration. This was shown to provide a good first order correction to optically derived SFRs, while smaller differences still remain between different SFR indicators that are likely to be related to different star formation histories and/or extinction properties (Sullivan et al. 2001).

The above studies were based on the comparison of different SFR indicators for samples of relatively low redshift galaxies, selected at optical or UV wavelengths, which are prone to dust induced biases. Furthermore, at intermediate redshifts where the $\mathrm{H} \alpha$ line falls out of the optical window and the $[\mathrm{OII}] \lambda 3727$ line can instead be used to measure the SFR $(0.3<z<0.8)$, a direct comparison is more difficult (see Cardiel et al. 2003). In this paper we explore the validity of the luminosity- (or SFR) dependent obscuration at both low $(z \lesssim 0.3)$ and intermediate $(0.3<z<0.8)$ redshifts, using a sample of star-forming galaxies selected at radio wavelengths (which are insensitive to dust obscuration) with spectroscopic information.
Throughout this paper we adopt $H_{0}=70$ $\mathrm{km} \mathrm{s}^{-1} \mathrm{Mpc}^{-1}, \Omega_{M}=0.3$, and $\Omega_{\Lambda}=0.7$.

\section{OBSERVATIONS}

The Phoenix Deep Survey (PDS) includes a $1.4 \mathrm{GHz}$ survey made using the Australia Telescope Compact Array (ATCA) covering a field a little over 4.5 square degrees, selected to lie in a region of low optical obscuration and devoid of bright radio sources (Hopkins et al. 2003a, and references therein). Optical imaging at the Anglo-Australian Telescope (AAT) has produced an optical catalogue probing to $R=22.5$ for the whole field, and $2 \mathrm{dF}$ multi-object spectroscopy from the AAT provides spectra for many of the optically identified radio sources (Georgakakis et al. 1999; Afonso 2002). The spectra were taken using the low resolution gratings $270 \mathrm{R}, 316 \mathrm{R}$ and 300B. Most objects were observed through only one of the red or blue gratings, with a small number being observed through both. The $270 \mathrm{R}$ and $316 \mathrm{R}$ gratings provide a wavelength coverage of $5000 \lesssim \lambda \lesssim 8500 \AA$, and the $300 \mathrm{~B}$ gives $4000 \lesssim \lambda \lesssim 7000 \AA$. Redshifts were determined by visual inspection of the spectra, and line parameters were measured through Gaussian fitting using the SPLOT package in IRAF. There are currently a total of 445 galaxies with measured spectra, of which 138 were securely classified as star forming using optical emission line diagnostic diagrams. A more detailed account of the spectroscopic data reduction and classification can be found elsewhere (Georgakakis et al. 1999; Afonso 2002).

\section{STAR FORMATION RATES IN THE FAINT RADIO} POPULATION

${ }^{1}$ CAAUL, Observatório Astronómico de Lisboa, Tapada da Ajuda, 1349-018 Lisboa

${ }^{2}$ Onsala Space Observatory, S-43992 Onsala, Sweden

3 email: jafonso@oal.ul.pt

${ }^{4}$ Department of Physics and Astronomy, University of Pittsburgh, 3941 O'Hara Street, Pittsburgh, PA 15260, USA; ahopkins@phyast.pitt.edu

5 Hubble Fellow

${ }^{6}$ Space Telescope Science Institute, 3700 San Martin Drive, Baltimore MD 21218, USA; b.mobasher@stsci.edu

7 Also affiliated to the Space Sciences Department of the European Space Agency

8 email: cesario@oal.ul.pt 
The origin of $1.4 \mathrm{GHz}$ emission in star-forming galaxies is primarily thought to be synchrotron radiation from relativistic electrons, accelerated by the shocks from supernova ejecta. The insensitivity of radio wavelengths to dust obscuration, makes radio emission a particularly attractive way of estimating SFRs in star-forming galaxies. The relation between SFR and $1.4 \mathrm{GHz}$ luminosity can be written as

$$
\mathrm{SFR}_{1.4 \mathrm{GHz}}=\frac{L_{1.4 \mathrm{GHz}}}{8.4 \times 10^{20} \mathrm{~W} \mathrm{~Hz}^{-1}} \mathrm{M}_{\odot} \mathrm{yr}^{-1}
$$

for a Salpeter IMF with stellar masses between 0.1 $100 \mathrm{M}_{\odot}$ (Haarsma et al. 2000). Using this relation, and calculating $L_{1.4 \mathrm{GHz}}$ assuming a radio spectral index $\alpha$ of $0.8\left(\mathrm{~S}_{\nu} \propto \nu^{-\alpha}\right)$, characteristic of synchrotron radiation, the SFRs for the star-forming galaxies in the Phoenix spectroscopic sample were calculated and are presented in Figure 1. The depth and area of the radio survey and the spectroscopic follow-up allow sampling star-formation rates from below one to a few hundred solar masses per year.

Of particular interest is the comparison between the SFRs as given by the radio and the optical line emission. To calculate the SFR from $\mathrm{H} \alpha$, the conversion indicated by Kennicutt (1998), for an IMF and mass limits as for equation (1), is used

$$
\mathrm{SFR}_{\mathrm{H} \alpha}=\frac{L_{\mathrm{H} \alpha}}{1.27 \times 10^{34} \mathrm{~W}} \mathrm{M}_{\odot} \mathrm{yr}^{-1} .
$$

The choice of a different IMF would change equations (1) and (2) by a similar factor (which for the most commonly used IMFs would be $\sim 2-4$ ), since both SFR indicators are sensitive to stars in roughly the same mass range $\left(\mathrm{M} \gtrsim 8 \mathrm{M}_{\odot}\right)$. Hence, a comparison between the SFRs derived from radio and $\mathrm{H} \alpha$ emission will be insensitive to the particular IMF shape choosen.

For galaxies at higher redshift $(z \gtrsim 0.3)$, where $\mathrm{H} \alpha$ could not be measured, one can use the [OII] $\lambda 3727$ flux. This method relies on equation (2) and the observed ratio between [OII] and $\mathrm{H} \alpha$. A value of $F_{[\mathrm{OII}]}=0.45 \times F_{\mathrm{H} \alpha}$ (Kennicutt 1998) is commonly used. However, using the first data release of the Sloan Digital Sky Survey (Abazajian et al. 2003), Hopkins et al. (2003b) observes a median relation for radio-detected star-forming galaxies of $F_{\text {[OII }]}=0.23 \times F_{\mathrm{H} \alpha}$ (with a scatter of around 0.1 ), finding the difference to be due to the incompleteness of previous samples. The range of optical luminosities sampled by Hopkins et al. (2003b) is similar to the one in the present Phoenix spectroscopic sample $\left(M_{R} \sim 20-23.5\right)$ and is not enough to reveal the optical luminosity dependence in the $[\mathrm{OII}] / \mathrm{Ha}$ ratio observed by Jansen, Franx \& Fabricant (2001), that produces lower [OII]/Ha ratios for galaxies with higher optical luminosities. Also, the range of extinctions, as given by the Balmer decrement, sampled by the Hopkins et al. (2003b) sample $(\mathrm{H} \alpha / \mathrm{H} \beta$, between 3 and 12 ) is similar to that of the present work (as will be seen below). We thus adopt the determination of Hopkins et al. (2003b) to convert our measured [OII] luminosities to $\mathrm{H} \alpha$ values, using equation (2) to obtain the corresponding SFR estimate.

Figure 2 compares the SFRs derived from the radio luminosity and line emission. Although a correlation exists, as expected, the SFRs derived from $1.4 \mathrm{GHz}$ are in general higher that those calculated from nebular lines, especially for higher luminosities (or SFRs). This effect, seen previously in several studies (Cram et al. 1998; Hopkins et al. 2001; Sullivan et al. 2001), is attributed to dust obscuration, which affects the optical line emission. Furthermore, one can conceive an amount of obscuration which increases with the SFR (a SFR-dependent dust obscuration). This has been explored with considerable success for nearby optical (Hopkins et al. 2001) and UV selected galaxies (Sullivan et al. 2001).

\section{SFR-DEPENDENT DUST OBSCURATION}

For a subset of the Phoenix sample described above, an estimate of the extinction can be made from the observed Balmer decrement $(\mathrm{H} \alpha / \mathrm{H} \beta)$. Stellar absorption of the Balmer lines was corrected by assuming an average value of $2 \AA$ for the equivalent width $(\mathrm{EW})$ of the $\mathrm{H} \beta$ absorption in star-forming galaxies (Tresse et al. 1996; Georgakakis et al. 1999), with a similar value (2.1 $\AA$ ) being used for the $\mathrm{H} \alpha$ line (equation (2) of Miller \& Owen 2002). Figure 3 shows the resulting Balmer decrements, corrected for stellar absorption, as a function of SFR, derived from the radio luminosity using equation (1). Unlike previous studies (Hopkins et al. 2001; Sullivan et al. 2001), a tight correlation is not observed here. Rather, a trend for a broader range of obscurations for higher SFR systems is observed. This behaviour seems to be due to different selection criteria and small number statistics, as we now explain.

The sample used by Hopkins et al. (2001) to derive the relation between SFR and obscuration includes only nearby $(z \leq 0.03)$ galaxies with $\mathrm{EW}(\mathrm{H} \alpha)$ larger than $30 \AA$. While no clear trend is seen in the present sample when restricted to this EW value, the higher limit of $60 \AA$ (filled circles in Figure 3) does suggest a closer match to the observations in Hopkins et al. (2001).

On the other hand, Buat et al. (2002) also observed a dual behaviour: while nearby star forming galaxies behave similarly to what is present in Figure 3, a sample of IUE galaxies shows a much tighter correlation, as that observed for the UV-selected sample of Sullivan et al. (2001). This suggests that UV-selection results in some kind of bias that is avoided with the present sample. Completeness in Figure 3 is not easy to quantify, given the several selection criteria (initially the radio flux limit, followed by the optical identification and $2 \mathrm{dF}$ spectroscopy, which imposes a practical limit of $R \sim 20$ ). However, it is possible to try to understand a possible bias in a magnitude-limited UV selected sample, and at the same time to evaluate the improvement of the present work, as we now show.

The tight relation between dust-free UV emission and SFR can be used to evaluate which regions of Figure 3 are not accessible to a magnitude-limited UV study. Assuming a limiting magnitude $m_{\mathrm{UV}}=18.5$ (as in Sullivan et al. 2001), an intrinsic SFR (ie, $L_{\mathrm{UV}}$ before obscuration) and redshift will define the maximum value of the Balmer decrement that still allows a detection. Figure 4 shows the present sample, separated according to redshift, overlaid with the maximum detectable Balmer decrement at $z=0.05$ (dotted line), 0.1 (dashed line) and 0.2 (dotdashed line). The conversion between SFR and $L_{\mathrm{UV}}$ uses 
the calibration from Kennicutt (1998), while the extinction at $2000 \AA$ is derived from the Balmer decrement using the procedures of Calzetti et al. (2000). An estimate of the $K$-correction is obtained using an average colour of $m_{\mathrm{UV}}-b=-1.5$ (Milliard et al. 1992). It is clear that the present sample represents a significant improvement for $z>0.1$. In particular, many of the galaxies showing high Balmer decrement values in the present study would not be detected in a UV survey limited to $m_{\mathrm{UV}}=18.5$. Sample selection thus seem to be a major source of bias when trying to investigate the correlation between dust obscuration and SFRs.

Given the large scatter present in Figure 3, a SFRdependent reddening correction is obviously unsuitable for application in galaxies where a direct estimate of obscuration exists. However, a trend for higher average Balmer decrement (and greater distribution width) with increasing SFR seems to exist. This can still be useful as a preliminary dust obscuration estimate for large samples of galaxies where no other measure of obscuration is available. Although in practice the form of the derived relation may be comparable to the ones in Hopkins et al. (2001) and Sullivan et al. (2001), here we recognize that there is no tight correlation between obscuration and SFR, but an average obscuration may still be defined for any given SFR. As can be seen in Figure 3 the resulting correction will be affected by large uncertainties for individual galaxies, especially at large SFRs.

The sample was thus split into 7 bins of $\log (\mathrm{SFR})$ (as estimated from the radio luminosity), each having between 5 and 16 objects. The median $\log$ (SFR) and Balmer decrement in each bin were then found (shown as asterisks in Figure 3). A linear fit, taking into account the errors in both quantities, results in

$$
\left(\frac{\mathrm{H} \alpha}{\mathrm{H} \beta}\right)_{\text {median }}=1.29 \log (\mathrm{SFR})+5.06,
$$

with a correlation coefficient of 0.8 . Keeping in mind the meaning and limitations of this correlation, as seen in Figure 3 , one can now test its usefulness as a first correction for the effect seen in Figure 2.

The departure of the observed Balmer decrement from the Case B value of 2.86 (e.g., Brocklehurst 1971), can be related to the color excess for nebular emission lines, $E(B-V)_{\text {gas }}$, and extinction, $k(\lambda)$, by

$$
\left(\frac{\mathrm{H} \alpha}{\mathrm{H} \beta}\right)_{\text {Case B }}=\left(\frac{\mathrm{H} \alpha}{\mathrm{H} \beta}\right)_{\text {obs }} 10^{0.4 E(B-V)_{\text {gas }}\left(k_{\alpha}-k_{\beta}\right)} .
$$

Substituting (3) into (4) gives a relation for the color excess as a function of SFR:

$$
E(B-V)_{g a s}=\frac{2.5}{\left(k_{\alpha}-k_{\beta}\right)} \log \left(\frac{2.86}{1.29 \log (\mathrm{SFR})+5.06}\right) .
$$

Together with an appropriate extinction curve (the standard Galactic extinction curve of Cardelli, Clayton \& Mathis (1989) with $R_{V}=3.1$, found by Calzetti (2001) to describe well the reddening of the ionized gas in starforming galaxies), this can then be used to correct $L_{\mathrm{H} \alpha}$, and consequently, $\mathrm{SFR}_{\mathrm{H} \alpha}$, for dust obscuration:

$$
L_{\mathrm{H} \alpha}=L_{\mathrm{H} \alpha}^{o b s} 10^{0.4 E(B-V)_{g a s} k_{\alpha}} .
$$

where $L_{\mathrm{H} \alpha}^{o b s}$ can either be the observed $\mathrm{H} \alpha$ luminosity or the "effective" $\mathrm{H} \alpha$ luminosity derived from an observed [OII] flux.

Equation (5) gives the relation between extinction and the intrinsic SFR. Assuming this to be the value given by the radio luminosity could be a good approximation, but would create an artificial dependence between the corrected $\mathrm{H} \alpha \mathrm{SFR}$ and the one from $1.4 \mathrm{GHz}$. Instead, since the form for the SFR-dependent obscuration is monotonically increasing, an iteration over possible values for intrinsic SFR and the corresponding obscuration can be performed until the calculated obscured SFR converges with the observed value (Hopkins et al. 2001). We note that this procedure does not take into account any absorption of ionizing photons by dust inside HII regions. Charlot et al. (2002), modeling the observed spectra in non-Seyfert galaxies, estimate that this mechanism is responsible for the loss of $\sim 20 \%$ of ionizing photons. Given the large uncertainty associated with this value, however, we do not attempt any correction, noting that its magnitude would not significantly affect our results.

Figure 5 shows the resulting dust corrected relation for the SFR from line and radio luminosities. It is clear that the SFR-dependent dust absorption, while being a very coarse approximation, can successfully account for the first order offset between the SFRs derived from $\mathrm{H} \alpha$ or [OII] and radio luminosities for galaxies spanning a broad range of redshifts (out to $z \approx 0.8$ ). This would not be possible if the relations between Balmer decrements and SFR drawn from previous samples (Hopkins et al. 2001; Sullivan et al. 2001) had been used. The scatter still present has an rms of 0.4 dex about the best fit line, maintained from the scatter in Figure 2. The lack of an improvement lies in the coarse relationship between SFR and obscuration seen in Figure 3 - the linear fit to the median values cannot correct for the range of obscurations seen at each SFR.

There will be, of course, additional uncorrelated mechanisms involved in the $\mathrm{H} \alpha$ and radio emission which contribute to the scatter seen, but their quantification will only be possible after a precise account of the obscuration for each individual galaxy.

\section{SUMMARY}

A radio selected sample of star forming galaxies to $z \approx 0.8$ has been compiled from the Phoenix Deep Survey. The use of radio selection minimises bias in the sample due to dust obscuration effects. The relationship between obscuration and SFR is shown to be only for a higher Balmer decrement range at higher SFRs, contrary to the tight correlation observed in previous studies. Still, the use of a linear relation, reflecting only the broadest trend, was explored as a first order correction for large samples of galaxies with no direct measurement of obscuration. This successfully accounts for the major discrepancy between optical emission line SFR estimates and $1.4 \mathrm{GHz}$ luminosity estimates for all galaxies in the broad redshift range probed. However, a much more detailed correction of the dust obscuration is necessary for the study of the uncorrelated mechanisms (e.g., star formation histories) responsible for the scatter still present. 
We thank M. Sullivan for his comments and advice. JA gratefully acknowledges the support from the Science and Technology Foundation (FCT, Portugal) through the fellowship BPD-5535-2001 and the research grant ESO-FNU-43805-2001. AMH acknowledges support provided by the National Aeronautics and Space Adminis- tration (NASA) through Hubble Fellowship grant HSTHF-01140.01-A awarded by the Space Telescope Science Institute (STScI). JA dedicates this work to the memory of Gustavo Camejo Rodrigues, who will always be dearly remembered.

\section{REFERENCES}

Abazajian et al. 2003, AJ (submitted; astro-ph/0305492)

Afonso, J. 2002, PhD Thesis, University of London

Bell, E. F. 2003, ApJ, 586, 794

Brocklehurst, M. 1971, MNRAS, 153, 471

Buat, V., Boselli, A., Gavazzi, G., \& Bonfanti, C. 2002, A\&A, 383, 801

Calzetti, D., Armus, L., Bohlin, R., Kinney, A., Koornneef, J., \& Storchi-Bergmann, T. 2000, ApJ, 533, 682

Calzetti, D. 2001, PASP, 113, 1449

Cardelli, J. A., Clayton, G. C., \& Mathis, J. S. 1989, ApJ, 345, 245

Cardiel, N., Elbaz, D., Schiavon, R. P., Willmer, C. N. A., Koo,

D. C., Phillips, A. C., \& Gallego, J. 2003, ApJ, 584, 76

Charlot, S., Kauffmann, G., Longhetti, M., Tresse, L., White, S. D. M., Maddox, S. J., \& Fall, S. M. 2002, MNRAS, 330, 876

Cram, L., Hopkins, A., Mobasher, B., \& Rowan-Robinson, M. 1998, ApJ, 507, 155

Genzel, R. \& Cesarsky, C. J. 2000, ARA\&A, 38, 761

Georgakakis, A., Mobasher, B., Cram, L., Hopkins, A., Lidman, C., \& Rowan-Robinson, M. 1999, MNRAS, 306, 708

Haarsma, D. B., Partridge, R. B., Windhorst, R. A., \& Richards, E. A. 2000, ApJ, 544, 641
Hopkins, A. M., Mobasher, B., Cram, L., \& Rowan-Robinson, M. 1998, MNRAS, 296, 839

Hopkins, A., Connolly, A., Haarsma, D., \& Cram, L. 2001, AJ, 122, 288

Hopkins, A. M., Afonso, J., Chan, B., Cram., L. E., Georgakakis, A., \& Mobasher, B. 2003a, AJ, 125, 465

Hopkins, A. M., Miller, C. J., Nichol, R. C., Gómex, P. L., Goto, T., Connolly, A. J., Bernardi, M. \& Tremonti, C. A. 2003b, AJ, submitted

Ivison, R. J., Smail, I., Barger, A. J., Kneib, J.-P., Blain, A. W., Owen, F. N., Kerr, T. H., \& Cowie, L. L. 2000, MNRAS, 315, 209

Jansen, R. A., Franx, M., \& Fabricant, D. 2001, ApJ, 551, 825

Kennicutt, R. C., Jr. 1998, ARA\&A, 36, 189

Miller, N. A. \& Owen, F. N. 2002, AJ, 124, 2453

Milliard, B., Donas, J., Laget, M., Armand, C., \& Vuillemin, A. 1992, A\&A, 257, 24

Smail, I., Ivison, R. J., Blain, A. W., \& Kneib, J.-P. 2002, MNRAS, 331,495

Sullivan, M., Mobasher, B., Chan, B., Cram, L., Ellis, R., Treyer, M., \& Hopkins, A. 2001, ApJ, 558,72

Tresse, L., Rola, C., Hammer, F., Stasinska, G., Le Fevre, O., Lilly, S. J., \& Crampton, D. 1996, MNRAS, 281, 847 


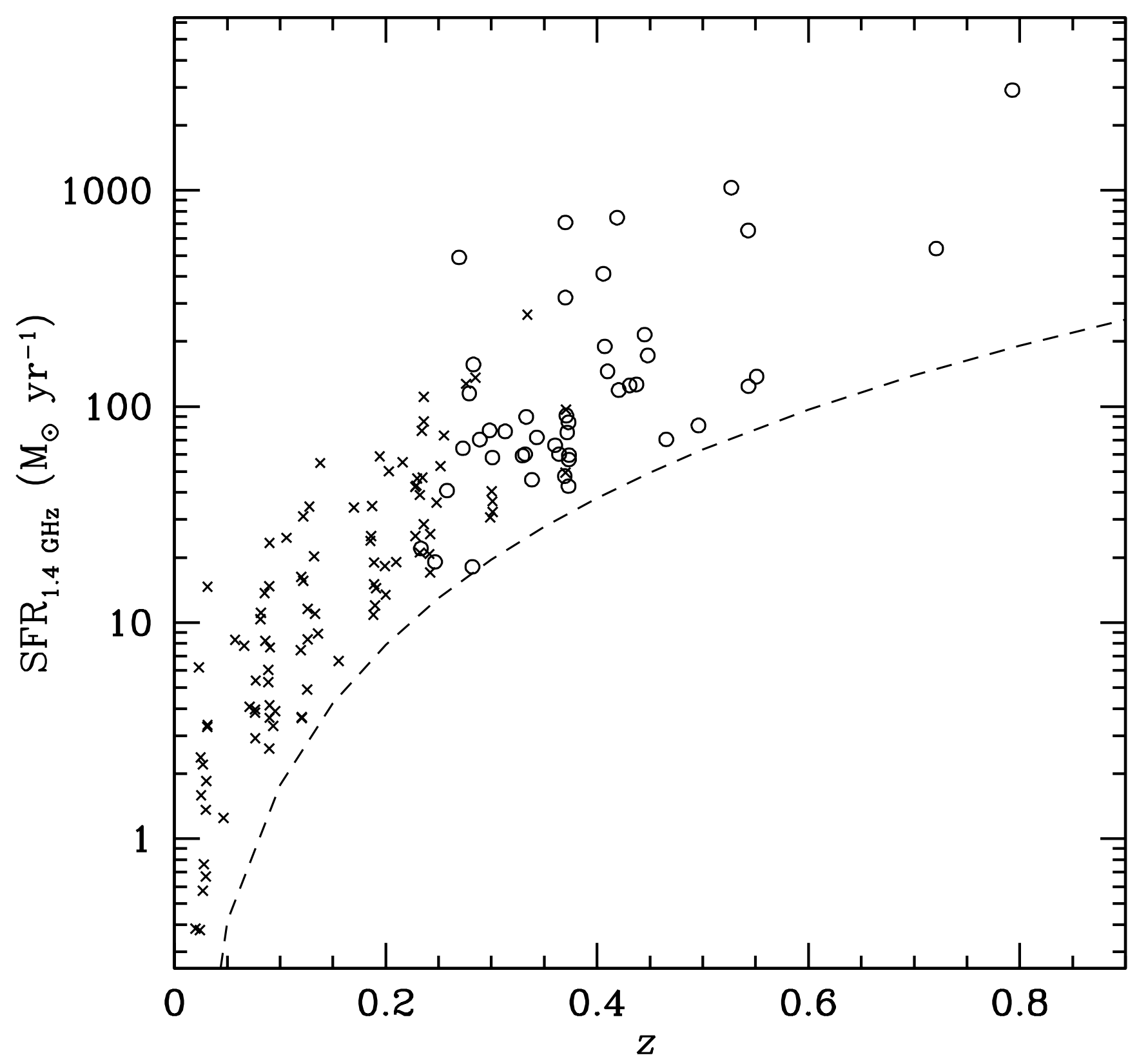

FIG. 1.- SFRs for star-forming galaxies in the Phoenix Deep Survey, as estimated from their radio luminosity. Crosses indicate galaxies with an $\mathrm{H} \alpha$ measurement suitable for a SFR estimate, while circles denote those where only [OII] $\lambda 3727$ is measured. The dashed line indicates the $60 \mu \mathrm{Jy}$ detection limit of the radio observations. 


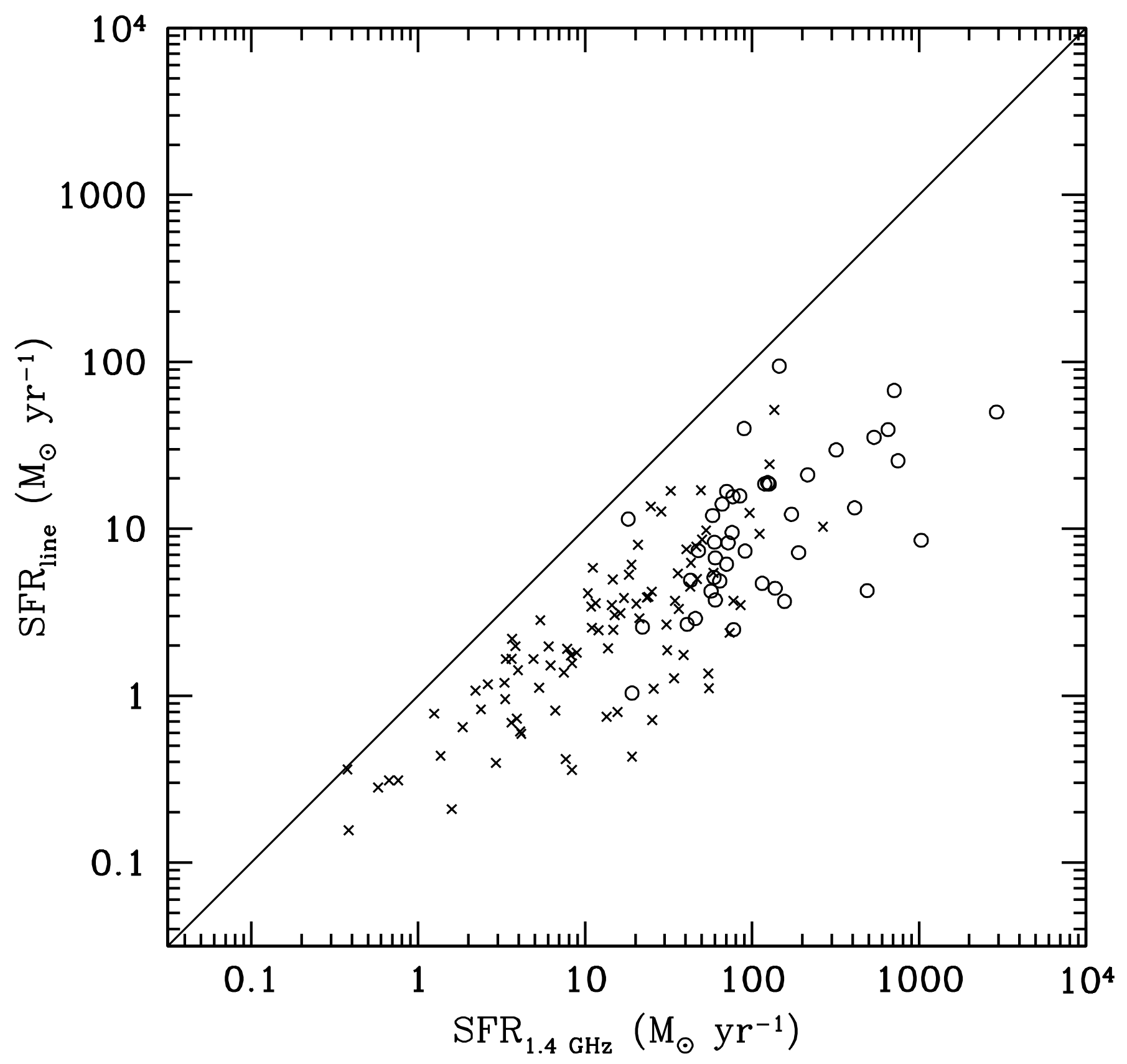

FIG. 2.- Comparison between the SFRs derived from $1.4 \mathrm{GHz}$ and from $\mathrm{H} \alpha$ or [OII] luminosities. The crosses represent galaxies with direct $\mathrm{H} \alpha$ measurement, circles denote galaxies at higher redshift, where [OII] was used as a proxy for the $\mathrm{H} \alpha$ line intensity. 


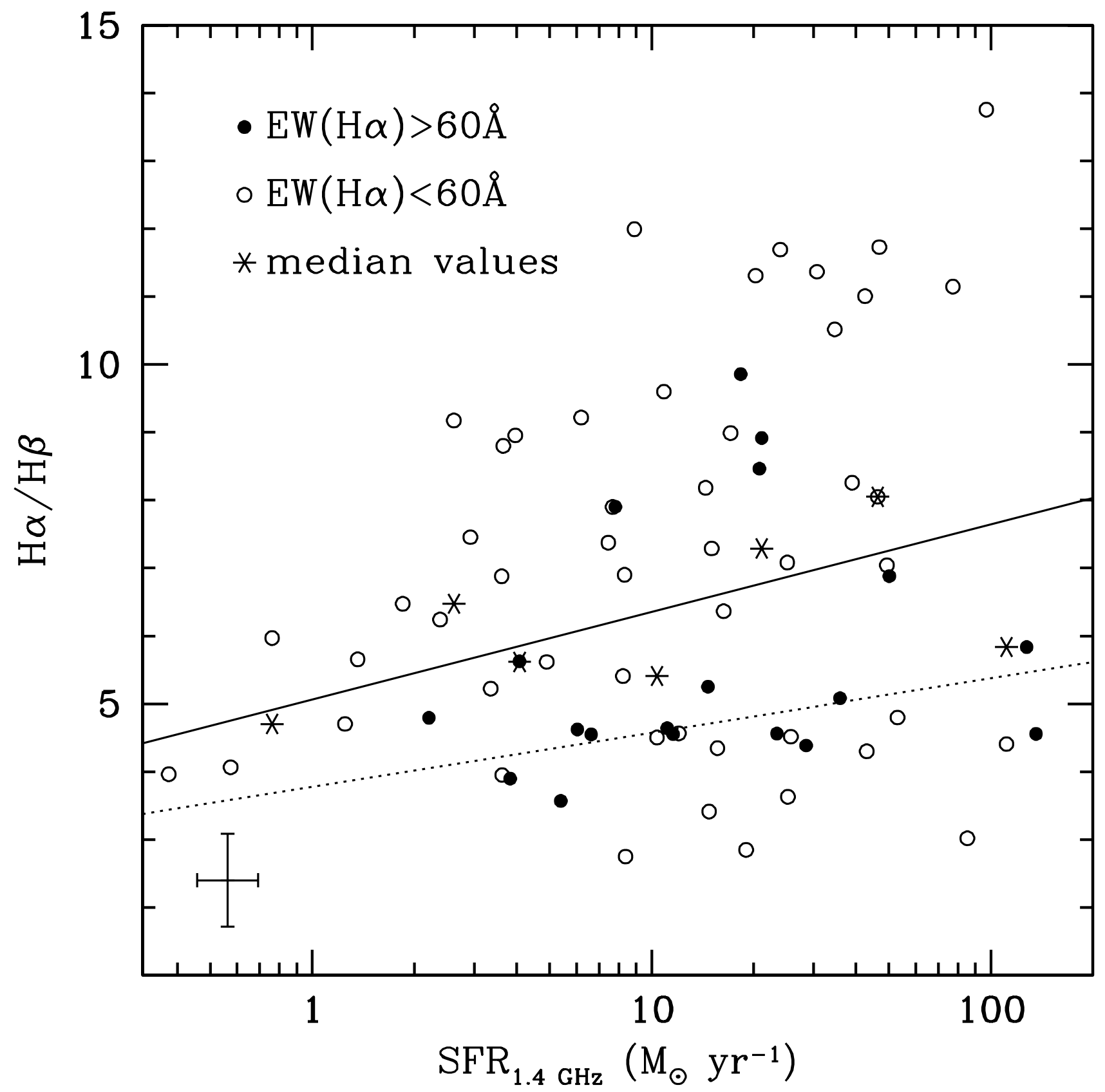

FIG. 3.- Balmer decrements, corrected for stellar absorption, as a function of SFR derived from radio luminosities. The solid line indicates the best fit to the median values (asterisks) in bins of $\log (\mathrm{SFR})$, while the dotted line shows the relationship derived in Hopkins et al. (2001) for a sample of local galaxies. The error bar in the bottom left corner shows the median errors for the Balmer decrement and radio flux measurements. 


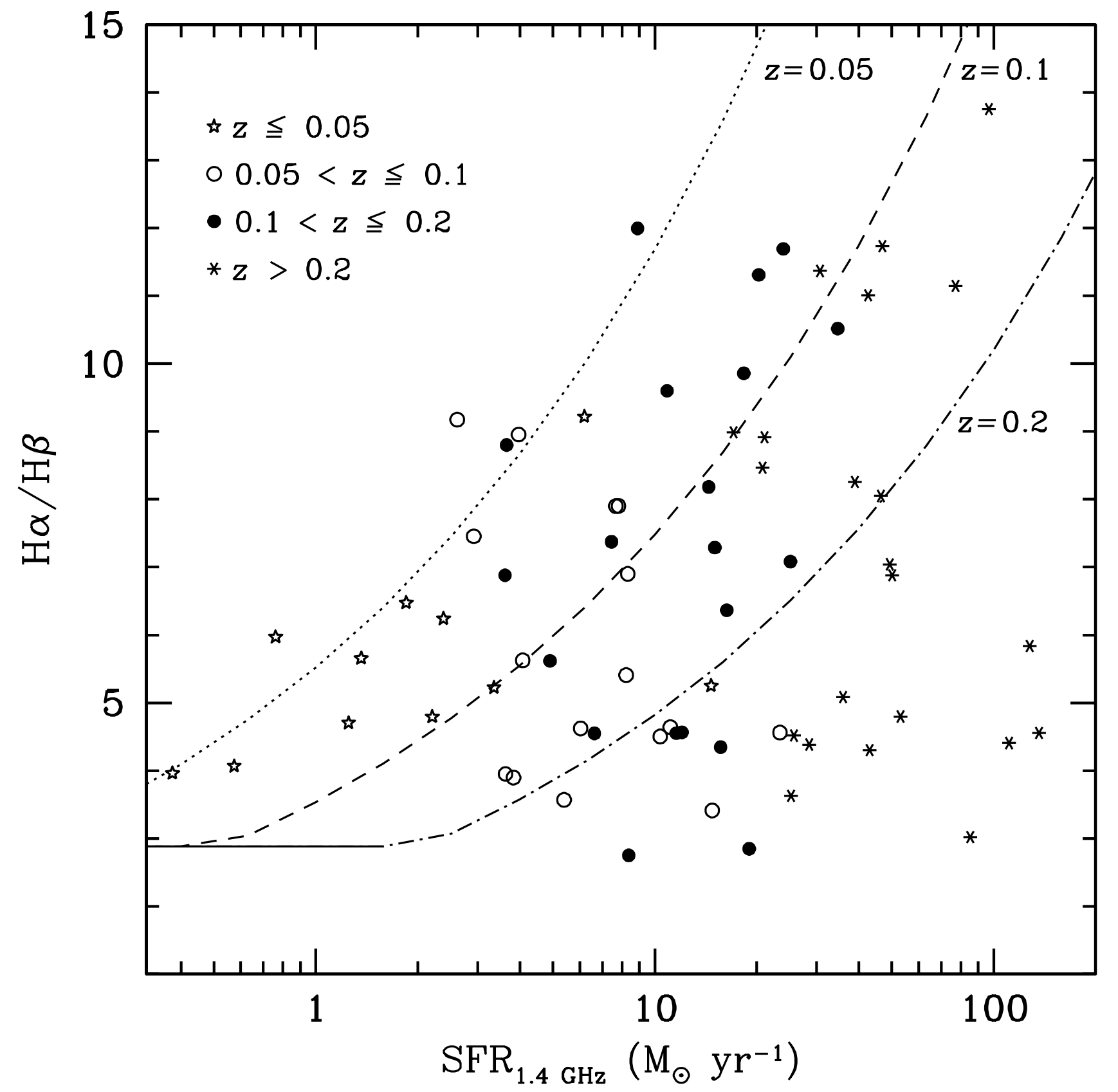

FIG. 4.- Data as in Fig. 3, marked according to redshift. The lines indicate the maximum Balmer decrement detectable for a given SFR at redshifts of $0.05,0.1$ and 0.2 , in a UV survey with a limiting magnitude of $m_{\mathrm{UV}}=18.5$. It is clear that most of the galaxies detected here displaying the highest Balmer decrements, would not be detected in such a UV survey. 


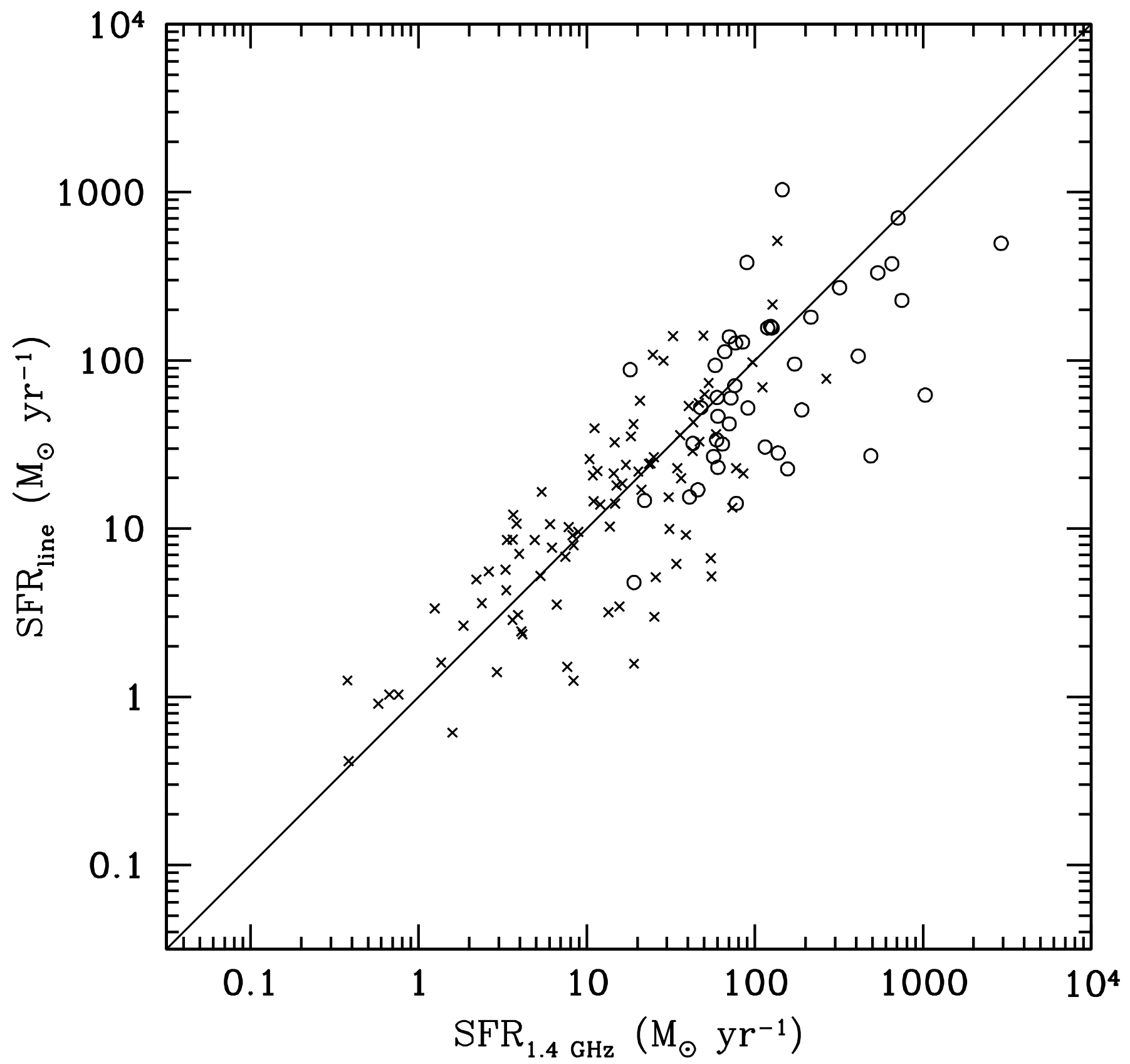

Fig. 5.- Same as Fig. 2, but with line luminosities corrected for dust obscuration using Equation (6). 